\title{
Three dimensional Conformal Field Theories from Sasakian seven-manifolds*
}

\author{
D. Fabbri \\ Dipartimento di Fisica Teorica, Università di Torino, \\ via P. Giuria 1, I-10125 Torino.
}

ABSTRACT: We present the construction of the candidate conformal field theories dual to $A d S_{4}$ non-maximally supersymmetric compactifications of 11D supergravity. We compare the spectra of the two theories and discuss the realization of the baryonic symmetries. Finally we comment the presence in the spectrum of long multiplets with rational energies, trying to explain their existence.

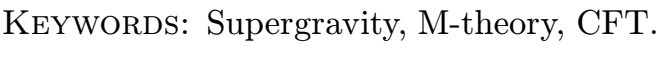

\section{Introduction}

One of the most exciting aspects of string theory that has emerged over the past decade is the deep interplay between geometry and physics.

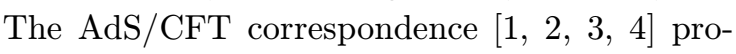
vides us the possibility of testing the connection between some geometrical features of the supergravity (SUGRA) compactifying manifold and the field content of the dual superconformal field theory (SCFT).

The purpose of [in , on which this talk is based, is twofold. On one side we intend to identify the gauge theory living on the boundary of $A d S_{4}$, whose IR fixed point should realize the SCFT dual to 11D SUGRA compactified on $A d S_{4} \times$ $(G / H)^{7}$, where $(G / H)^{7}$ is a homogeneous seven manifold. On the other side, we want to check the correspondence by comparing the spectra of the two theories.

Especially in the case of M-theory, where we have no deep control on the fundamental dynamics, the geometrical hints are essentially the only guidelines that help us in the construction of the worldvolume theory of a collection of branes placed at a conifold singularity in transverse space, namely the vertex of the metric cone over $(G / H)^{7}$. We will see that when this cone, $\mathcal{C}(G / H)$, admits a toric description, it is possible to argue

\footnotetext{
*Talk presented at the TMR conference in Paris, September 1999.
}

the gauge group and the matter field content of this theory.

We are interested in the $\mathcal{N} \geq 2$ supersymmetric compactifications, which were classified in $1984[6]$. The possible internal manifolds are the sasakian [ī] and trisasakian cosets:

$$
\begin{aligned}
M^{111} & \equiv \frac{S U(3) \times S U(2) \times U(1)}{S U(2) \times U(1) \times U(1)} \\
Q^{111} & \equiv \frac{S U(2) \times S U(2) \times U(2)}{U(1) \times U(1)} \\
V_{5,2} & \frac{S O(5)}{S O(3)} \\
N^{010} & \frac{S U(3)}{U(1)}
\end{aligned} \rightarrow \mathcal{N}=2
$$

Contrary to the type IIB case of N D3-branes at a conifold singularity [is a nontrivial superpotential provides a constraint on the observable operators of the boundary SCFT, for the gauge theory of N M2-branes, relevant to this work, it seems to be no such a superpotential to help us in the identification of the effective degrees of freedom at the strong coupling conformal point.

Despite this lack, once again geometry comes in our aid. Indeed it is possible to recognize a common algebraic structure between the pattern of the KK short multiplets and the description of the transverse cone $\mathcal{C}(G / H)$ as an algebraic 
(sub)manifold. Relying on this analogy, it is possible to associate to the generators of the polynomial ring of $\mathcal{C}(G / H)$ particular combinations of fundamental fields of the gauge theory. These combinations seem to be the effective degrees of freedom of the CFT, generating the chiral ring of conformal chiral operators, at the base of the spectrum.

In this way we are able to identify the CFT boundary operators dual to all the bulk short multiplets, matching not only the conformal dimensions of the first with the energies of the latter, but even the flavor and $R$-symmetry quantum numbers of each couple. In this sense, the richer structure of the spectra of non-maximally supersymmetric theories guarantees a less trivial test of duality than in the case of spherical compactifications.

One further highly non-trivial check is given by the SUGRA predictions on the anomalous conformal dimensions of the fundamental fields of the boundary theory. These predictions are based on the global baryonic symmetries of the latter $\left[2 \overline{0}_{0}^{1}, 2 \overline{2} \overline{1}\right]$, which reflect the existence of nontrivial homology cycles of the internal $(G / H)^{7}$ [2그.

Finally, an interesting aspect of these nonmaximally supersymmetric AdS/CFT pairs, seems to be the existence of some intriguing quantum mechanism that prevents certain long multiplets of the spectrum from acquiring anomalous dimensions. On the base of a simple consideration on the spectra of different supersymmetric compactifications, we will try to hint a possible direction towards the solution of the puzzle.

\section{Identification of the gauge theory}

We want now to describe the fundamental steps in the search for the worldvolume thoery of $\mathrm{N}$ $M 2$-branes sitting at the vertex of $\mathcal{C}(G / H)$. We will focus on the cases where the cone is a toric manifold, namely for $(G / H)^{7}=M^{111}$ or $Q^{111}$. Hence we are looking for an $\mathcal{N}=2$ supersymmetric gauge theory in three dimensions, whose moduli space of vacua should reproduce the one of the brane system. We make a minimality hypothesis about the matter field content: we assume that the fundamental fields, apart from the gauge bosons, transform in the most basic ${ }^{1}$ representation of the $O s p(\mathcal{N} \mid 4)$ superconformal group: the supersingleton, which has a field-theoretic realization as a particularly constrained chiral superfield.

The general structure of a three dimensional $\mathcal{N}=2$ supersymmetric gauge theory coupled to chiral matter is severly constrained. The only freedom we have is in the choice of

\section{- the gauge group;}

- the scalar Kähler manifold, namely (in the renormalizable flat case) the number and flavor representations of the matter chiral multiplets;

- the holomorphic superpotential.

Let us begin with identifying the abelian gauge theory living on a single brane. Being the gauge fields frozen at the conformal point $\left(g_{Y M}\right.$ is dimensionful), we can neglect the Coulomb branch and focus on the Higgs branch of the moduli space, parametrized by the vev of the scalars in the chiral multiplets. This branch should reproduce the space of vacua of the $M 2$-brane, which is the transverse space $\mathcal{C}(G / H)$.

This requirement is easily achieved when the cone admits a toric description. Roughly speaking, this means that $\mathcal{C}(G / H)$ can be viewed as the Kähler quotient:

$$
\mathcal{C}(G / H)=\frac{\mathbb{C}^{p}}{\left(\mathbb{C}^{*}\right)^{(p-4)}}
$$

for some $p \geq 4$. In this case, we can take as chiral fields the $p$ coordinates of $\mathbb{C}^{p}$. The flavor quantum numbers will be determined by the natural lifting on $\mathbb{C}^{p}$, of the isometries of $(G / H)^{7}$. By choosing as abelian gauge group the $U(1)^{(p-4)}$ compact part of the modding $\left(\mathbb{C}^{*}\right)^{(p-4)}$, we obtain as $D$-term equations its non-compact $\mathbb{R}^{+}(p-4)$ part, in such a way to reproduce (12.1) as the moduli space of vacua of the gauge theory.

Let us see how this construction is concretely implemented in the case where $(G / H)^{7}$ is the

\footnotetext{
${ }^{1}$ in the sense that all the higher representation are realizable as tensor products of this one.
} 
coset $M^{111}$, whose metric cone admits the following toric description:

$$
\mathcal{C}\left(M^{111}\right)=\frac{\mathbb{C}^{5}}{\mathbb{C}^{*}} .
$$

The quotient is defined by the equivalence:

$$
\left(U^{i}, V^{A}\right) \sim\left(\lambda^{2} U^{i}, \lambda^{-3} V^{A}\right), \quad \lambda \in \mathbb{C}^{*},
$$

where $U^{i}$ and $V^{A}(i=1,2,3, A=1,2)$ are the five coordinates of $\mathbb{C}^{5}$, transforming respectively in the fundamental representation of the $S U(3)$ and of the $S U(2)$ flavor factors. The $\mathbb{R}^{+} \subset \mathbb{C}^{*}$ modding action can be fixed by imposing:

$$
\sum_{i}\left|U^{i}\right|^{2}=\sum_{A}\left|V^{A}\right|^{2}
$$

while the $U(1)$ part of $(2.3 i)$ determines the charges of the corresponding chiral fields ( 2 and -3 respectively).

With this choice of gauge group and chiral fields, the $D$-term of the bosonic potential is given by:

$$
\mathcal{U}(z, \bar{z})=\left(\sum_{i}\left|u^{i}\right|^{2}-\sum_{A}\left|v^{A}\right|^{2}\right)^{2}
$$

whose minimization, together with the gauge equivalence:

$$
\left(u^{i}, v^{A}\right) \sim\left(e^{2 i \theta} u^{i}, e^{-3 i \theta} v^{A}\right)
$$

exactly reproduces the equation of the cone (12.3i). For symmetry reasons, it is worth to introduce a second $U(1)$ group, yielding the following couples of charges for the fundamental supersingletons:

$$
\left\{\begin{array}{c}
U^{i}:(2,-2) \\
V^{A}:(-3,3)
\end{array} .\right.
$$

The diagonal factor will actually decouple, leaving the gauge group $U(1)^{2} / U(1)_{\text {diagonal }}$.

The non-abelian generalization of this gauge theory is easily obtained by promoting the $U(1)$ gauge groups to $S U(N)$, with chiral matter in the following color representations:

$$
\left\{\begin{array}{c}
U^{i}: \mathbf{N}^{\otimes_{\mathrm{s}} 2} \otimes \overline{\mathbf{N}}^{\otimes_{\mathrm{s}} 2} \\
V^{A}: \overline{\mathbf{N}}^{\otimes_{s} 3} \otimes \mathbf{N}^{\otimes_{\mathrm{s}} 3}
\end{array}\right.
$$

\section{The conformal theory}

Let us now consider the observable fields at the IR fixed point of this theory, where the gauge fields are integrated out. First of all they will reduce to the gauge-invariant composite operators, whose smallest holomorphic combination (in the abelian case) is given by

$$
X^{i j k A B} \equiv U^{i} U^{j} U^{k} V^{A} V^{B},
$$

transforming in the $(\mathbf{1 0}, \mathbf{3})$ of $S U(3) \times S U(2)$.

An alternative description of $\mathcal{C}\left(M^{111}\right)$ is in terms of an algebraic submanifold of $\mathbf{C}^{30}$, where the complex space can be parametrized by the $10 \times 3=30 X^{i j k A B}$ of (3.1). The embedding is given by 325 quadratic homogeneous equations in these coordinates $X$. In other words, on the defining locus of the cone certain quadratic combinations of the $X^{i j k A B}$ identically vanish. This fact is quite general and reveals a deep connection between algebraic geometry and representation theory. Without entering into mathematical details, it is worth to stress that the embedding equations are equivalent to putting to zero certain irreducible representations spanned by the quadratic products of the $X$ 's. Indeed the symmetric tensor product:

$$
X^{i j k A B} \cdot X^{l m n C D} \leftrightarrow(\mathbf{1 0}, \mathbf{3}) \otimes_{\mathbf{s}}(\mathbf{1 0}, \mathbf{3})
$$

branches into several irreducible representations of the flavor group $S U(3) \times S U(2)$. Among these, only the highest weight survives, while the other constitute just the 325 combinations that, once equated to zero, yield the embedding equations of the cone.

In the $M^{111}$ case, the only surviving polynomials of homogeneous degree 6 and 4, respcetively in the $U^{\prime}$ 's and the $V$ 's, are those combinations belonging to the completely symmetric flavor representation:

$$
\square \square \square_{S U(3)} \otimes \square \square \square_{S U(2)} \cdot
$$

If we consider higher degree polynomials in the $X$ 's we find that, due to the vanishing of certain quadratic subfactors, the only surviving combinations are always those completely symmetrized in the flavor indices.

The mathematical structure underlying these selection rules is that of a ring. On one side we 
have the polynomial ring characterizing the cone $\mathcal{C}\left(M^{111}\right)$ as an algebraic manifold:

$$
\frac{\mathbf{C}\left[X^{i j k A B}\right]}{\mathcal{I}_{325}}
$$

where $\mathcal{I}_{325}$ is the ideal of the embedding equations. On the other side we have the isomorphic chiral ring of the holomorphic color singlet operator products, modded out by the proper ideal of vanishing relations.

This ring structure is easily extended to the non-abelian generalization of the gauge theory. Indeed it is possible to show that there is always only one possible way to contract the gauge indeces into a color singlet, for each homogeneous polynomial in the $X$ 's, completely flavor symmetrized.

It is important to remark the difference between our coset compactifications and the $T^{11}$ case of [i8]. While for the $Q^{111}$ and $M^{111}$ the non-highest weight part of (3.21) is given by a considerable set of equations (respectively 9 and 325 ), in the $T^{11}$ case the only vanishing quadratic combination of the $X$ 's corresponds to the singlet representation of the flavor group $S U(2) \times$ $S U(2)$ which, in the non-abelian case, turns out to be just the superpotential of the boundary theory. In the other $M$-theory cases, there is no way to combine all the non-highest weight pieces of $(3.2)$ into a single flavor invariant with the right dimensions for a superpotential. This is the fundamental reason why the vanishing relations, which select the observable operators at the conformal point, are to be found in a highly nonperturbative quantum mechanism, other then the minimization of the potential.

\section{Comparison with the KK spec- trum}

The first test of correspondence between the CFT and the compactified SUGRA is the matching of the spectra of the two theories.

The KK compactifications of 11D SUGRA were extensively studied in the first half of the 80 's. In particular, the coset space $M^{111}$ was

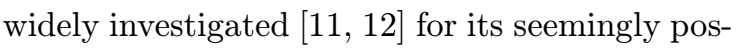
sibility to explain the origin of the $S U(3) \times S U(2) \times$ $U(1)$ gauge bosons of the standard model.
The developement of techniques such as harmonic analysis on coset spaces [1] $\left.{ }_{1}^{\prime}\right]$, allowed the computation of great part of the spectrum of these compactifications. But the problems concerning the fermionic spectrum (first of all the absence of chirality) and the occurence of the first string revolution rapidly distorted the attention from KK SUGRA.

Due to the renewed interest, in connection to the AdS/CFT conjecture, the KK spectra of the $M^{111}, Q^{111}$ and $V_{5,2}$ compactifications have been recently completed in a systematic way [1 $\left.1 \overline{4}_{4}^{1}, 1 \overline{1} \overline{5}_{1}^{\prime}\right]$.

We will focus in particular on the CFT operators of protected conformal dimensions, corresponding to KK states belonging to short representations of the $O s p(2 \mid 4)$ symmetry superalgebra. The following table lists all the different kinds of $B P S$-saturated representations and the supercovariant differential constraint implementing the corresponding shortening conditions on

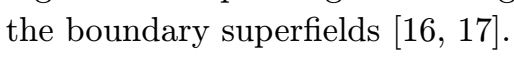

\begin{tabular}{|c|c|}
\hline multiplet & $\begin{array}{c}\text { differential constraint on } \\
\text { the boundary superfield }\end{array}$ \\
\hline short graviton & $\mathcal{D}^{+\alpha} \Phi_{(\alpha \beta)}\left(x, \theta^{ \pm}\right)=0$ \\
\hline short gravitino & $\mathcal{D}^{+\alpha} \Phi_{\alpha}\left(x, \theta^{ \pm}\right)=0$ \\
\hline short vector & $\mathcal{D}^{+\alpha} \mathcal{D}_{\alpha}^{+} \Phi\left(x, \theta^{ \pm}\right)=0$ \\
\hline hypermultiplet & $\mathcal{D}^{+\alpha} \Phi\left(x, \theta^{ \pm}\right)=0$ \\
\hline massless graviton & $\left\{\begin{array}{l}\mathcal{D}^{+\alpha} \Phi_{(\alpha \beta)}\left(x, \theta^{ \pm}\right)=0 \\
\mathcal{D}^{-\alpha} \Phi_{(\alpha \beta)}\left(x, \theta^{ \pm}\right)=0\end{array}\right.$ \\
\hline massless gravitino & $\left\{\begin{array}{l}\mathcal{D}^{+\alpha} \Phi_{\alpha}\left(x, \theta^{ \pm}\right)=0 \\
\mathcal{D}^{-\alpha} \Phi_{\alpha}\left(x, \theta^{ \pm}\right)=0\end{array}\right.$ \\
\hline massless vector & $\left\{\begin{array}{l}\mathcal{D}^{+\alpha} \mathcal{D}_{\alpha}^{+} \Phi\left(x, \theta^{ \pm}\right)=0 \\
\mathcal{D}^{-\alpha} \mathcal{D}_{\alpha}^{-} \Phi\left(x, \theta^{ \pm}\right)=0\end{array}\right.$ \\
\hline supersingleton & $\left\{\begin{array}{c}\mathcal{D}^{+\alpha} \Phi\left(x, \theta^{ \pm}\right)=0 \\
\mathcal{D}^{-\alpha} \mathcal{D}_{\alpha}^{-} \Phi\left(x, \theta^{ \pm}\right)=0\end{array}\right.$ \\
\hline
\end{tabular}

For space reasons, it is not possible here, to make a complete list of the protected conformal superfields corresponding to all the short multiplets of the KK spectrum. We will focus on the most meaningful.

As we anticipated, the chiral ring of the SCFT is given by the following composite operators:

$$
\operatorname{Tr}\left[\left(U^{3} V^{2}\right)^{k}\right],
$$

where, following the prescription given in the last section, the flavor indices are completely symmetrized, while the trace symbol implies the only 
possible contraction into a color-singlet, compatible with this flavor representation. These chiral superfields find a complete matching with the tower of hypermultiplets of the KK spectrum. The only constraint we have, is on the anomalous dimensions of the fundamental supersingletons, which we are not able to directly deduce from the CFT:

$$
2=\Delta\left[\operatorname{Tr}\left(U^{3} V^{2}\right)\right]=3 \Delta(U)+2 \Delta(V) .
$$

In the next section we will show how $\Delta(U)$ and $\Delta(V)$ can be obtained from SUGRA computations, confirming the validity of our conjecture on the dual CFT.

Other important superfields are the conserved supercurrents:

$$
S U(3) J_{j}^{i}=\operatorname{Tr}\left[U^{i} \bar{U}_{j}-\frac{1}{3} \delta^{i}{ }_{j} U^{k} \bar{U}_{k}\right]
$$

and

$$
{ }^{S U(2)} J_{B}^{A}=\operatorname{Tr}\left[\bar{V}^{A} V_{B}-\frac{1}{2} \delta_{B}^{A} \bar{V}^{C} V_{C}\right],
$$

that transform in the adjoint representation of the flavor groups, $S U(3)$ and $S U(2)$ respectively, and contain the massless vectors associated to these symmetries. Two sets of semiconserved currents, corresponding to short vector multiplets of the KK spectrum are given by composing (4.3) and (4.4) with the chiral fields (4.15).

Finally, two towers of KK short gravitinos are matched by the following spinorial superfields:

$\operatorname{Tr}\left[\left(U \bar{U}\left(\mathcal{D}_{\alpha}^{+} \bar{V} V\right)+\bar{V} V\left(\mathcal{D}_{\alpha}^{+} U \bar{U}\right)\right)_{j B}^{i A}\left(U^{3} V^{2}\right)^{k}\right]$

and

$$
\operatorname{Tr}\left[\left(U^{i} U^{j} U^{k} V^{A} \mathcal{D}_{\alpha}^{-} V^{B} \epsilon_{A B}\right)\left(U^{3} V^{2}\right)^{k}\right] .
$$

All these superfields and the other which we have not listed, perfectly fit the masses, $R$-symmetry charges and flavor quantum numbers of the corresponding KK short multiplets.

\section{The baryonic symmetries}

The next item we want to discuss is the presence in the boundary theory of barion-like conformal operators and their interpretetion in terms of non-perturbative bulk states.
Already in the gold years of KK SUGRA, it was noted [22] that the existence of non trivial homological cycles in the internal manyfold implies the presence of massless vectors in the spectrum of the compactified SUGRA, due to the reduction of higher degree form potential on these cycles. The corresponding Betti multiplets have been related, in the AdS/CFT perspective, to global baryonic symmetries of the dual gauge theory [200]"

In the specific case considered in this talk, it is found that the manyfold $M^{111}$ has non vanishing second and fifth Betti numbers: $b_{2}\left(M^{111}\right)=$ $b_{5}\left(M^{111}\right)=1$, implying a $U(1)$ baryonic symmetry. All the KK multiplets (and hence all the dual conformal operators) result discharged under this symmetry. But we have not analysed, so far, all the possible color singlet products of fundamental supersingletons at our disposal. Among these, we have the totally antisymmetrized ${ }^{2}$ (in the $S U(N)$ color indices) product of $N$ singletons of the same kind, that we will abbreviate $\operatorname{det}(U)$ and $\operatorname{det}(V)$. The masses of these operators grow like $N$ in the large $N$ limit. Hence their SUGRA duals have to be found among non-perturbative states. Indeed we find the existence in $M^{111}$ of two particular families of non trivial supersymmetric 5-cycles, over which a solitonic $M 5$-brane can be wrapped. The energy of these $M$-theory configurations, simply related to the volume of the cycles, has the same large $N$ behaviour. The exact coefficient in this linear expression can be used as a SUGRA prediction on the conformal anomalous dimension of the corresponding fundamental singleton. In this case we find:

$$
\begin{aligned}
\Delta[\operatorname{det}(U)] & =\frac{4 N}{9} \Longrightarrow \Delta(U)=\frac{4}{9} \\
\Delta[\operatorname{det}(V)] & =\frac{N}{3} \Longrightarrow \Delta(V)=\frac{1}{3}
\end{aligned}
$$

These numbers perfectly fit the only constraint (4.2) we have from the SCFT side:

$$
3 \Delta(U)+2 \Delta(V)=2 .
$$

Furthermore, even the flavor irreducible representation of these configurations (deducible from the action of the $M^{111}$ isometries on the cycles of

\footnotetext{
${ }^{2}$ implying total symmetrization in the flavor indices
} 
each family) perfectly agree with the flavor quantum numbers of the corresponding determinant operators, yielding a highly non trivial check of duality.

\section{Comments about the rational long multiplets}

An interesting feature common to both type IIB and 11D SUGRA coset compactifications, first noted in [1] $\left.{ }_{1}^{1}\right]$ for the $T^{11}$ space, is the presence in the KK spectrum of long multiplets with rational energies (see also the talk by G. Dall'Agata about this point). This fact does not seem to be a mere coincidence. Indeed it has been recognized [1 101$]$ that the dual conformal operators follow a precise pattern: they are products of two or more quantities separately protected that, nevertheless, have no a priori reason to be globally protected. The conformal dimension of these operator products, as it is deduced by the energy of the corresponding SUGRA states, is instead given by the naive sum of the dimensions of the single factors.

To make a concrete example, both the $M^{111}$ and $Q^{111}$ bulk spectra contain a tower of long graviton multiplets, associated to boundary vector superfields of the form:

$$
\Phi_{(\alpha \beta)} \sim T_{(\alpha \beta)} \times J \times \phi,
$$

where $T_{(\alpha \beta)}, J$ and $\phi$ are respectively the stress energy tensor, a conserved vector current and a chiral operator, and the dimension of $\Phi$ is precisely given by:

$$
\Delta(\Phi)=\Delta(T)+\Delta(J)+\Delta(\phi) .
$$

We have not yet a definitive explaination of the quantum mechanism that seems to protect these operators from acquiring anomalous dimensions. But a simple consideration has emerged while studying the $\mathcal{N}=3 S C F T$ corresponding to the SUGRA compactification on the trisasakian manifold $N^{010}$ [1' 1 i'].

From the analysis of the $O s p(3 \mid 4)$ supermultiplets and of their decomposition into $\mathcal{N}=2$ irreducible representations [1 $\left.1 \mathrm{~g}_{1}^{\prime}\right]$, one can realize that the short $\mathcal{N}=3$ multiplets always contain long multiplets of the lower superalgebra. Furthermore, from a careful identification of the corresponding boundary conformal operators, we have recognized for some of these the same structure of (6.1). Obviously, belonging to the same supermultiplet of the higher supersymmetry algebra, these long $\mathcal{N}=2$ multiplets have energies that differ from those of the other states only by (half)-integers. Hence they are necessarily rational, despite the fact that, from the $\mathcal{N}=2$ viewpoint, they are long.

This simple consideration seems to hint that the quantum protection mechanism previously advocated may be found in a residual form of higher supersymmetry that the SCFT could reflect, such as a spontaneous supersymmetry breaking.

Acknowledgements. I would like to thank all the authors of the paper $[\overline{15}]$, on which this talk is based and also P. Termonia for his collaboration in the preliminary results on harmonic analysis. This work is supported by the European Commission TMR programme ERBFMRX-CT96-0045.

\section{References}

[1] J.M. Maldacena, Adv. Theor. Math. Phys. 2 (1998) 231, inep-th/9711200!

[2] S.S. Gubser, I.R. Klebanov, A.M. Polyakov, Phys. Lett. B428 (1998) 105, 'hep-th/9802109'.

[3] E. Witten, Adv. Theor. Math. Phys. 2 (1998) 253, hep-th/9802150!

[4] O. Aharony, S.S. Gubser, J. Maldacena, H. Ooguri and Y. Oz, hep-th/9905111'.

[5] D. Fabbri, P. Frè, L. Gualtieri, C. Reina, A. Tomasiello, A. Zaffaroni, A. Zampa, to be published on Nucl. Phys., preprinthep-th/9907219.'

[6] L. Castellani, L.J. Romans, N.P. Warner, Nucl. Phys. B241 (1984) 429.

[7] B.S. Acharya, J.M. Figueroa-O'Farrill, C.M. Hull and B. Spence, Adv. Theor. Math. Phys. 2 (1999) 1249, hep-th/9808014; J.M. Figueroa-O'Farrill, Class. Quant. Grav. 16 (1999) 2043, ihep-th/9902066!

[8] I. Klebanov and E. Witten, Nucl. Phys. B536 (1999) 199, hep-th/9807080!

[9] S.S. Gubser, Phys. Rev. D59 (1999) 025006, hep-th/9807164.

[10] A. Ceresole, G. Dall'Agata, R. D'Auria and S. Ferrara, Phys. Rev D61 (2000), hep-th/9905226. 
[11] E. Witten, Nucl. Phys. B186, 412 (1981).

[12] L. Castellani, R. D' Auria and P. Frè, Nucl. Phys. B239, 60 (1984).

[13] L. Castellani, R. D' Auria and P. Frè, Supergravity and superstrings: A geometric perspective. Voll. 1-2, Singapore, Singapore: World Scientific (1991); A. Salam and J. Strathdee, Annals Phys. 141 (1982) 316.

[14] D. Fabbri, P. Frè, L. Gualtieri, P. Termonia, Nucl. Phys. B560 (1999) 617, ihep-th/9903036; D. Fabbri, P. Frè, P. Merlatti, unpublished.

[15] A. Ceresole, G. Dall'Agata, R. D'Auria and S. Ferrara, hep-th/9912107i

[16] S. Ferrara, lectures at the University of Torino (winter 1998), unpublished.

[17] D. Fabbri, P. Frè, L. Gualtieri, P. Temrmonia, Class. Quant. Grav. 17 (2000) 55, hep-th/9905134!

[18] M. Billò, D. Fabbri, P. Frè, P. Merlatti, C. Reina, A. Tomasiello, A. Zaffaroni and A. Zampa, work in progress.

[19] P. Frè, L. Gualtieri, P. Termonia, Phys. Lett. B471 (1999) 27, hhep-th/9909188.

[20] E. Witten, _ JHEP $\mathbf{9 8 0 7}$ (1998) 006, hep-th/9805112.

[21] S.S. Gubser and I. Klebanov, Phys. Rev D58, (1998) 125025, hep-th/9808075.

[22] R. D'Auria and P. Frè, Ann. Phys. 162 (1985) 372. 